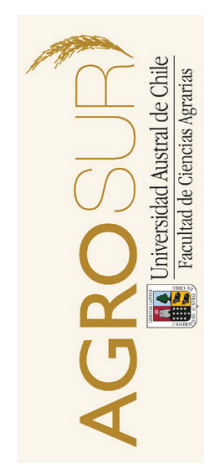

\section{Variación temporal del contenido de materia orgánica en dos suelos volcánicos bajo diferentes manejos agrícolas}

\author{
Temporal organic matter content variation on of two volcanic soils \\ under different agricultural managements
}

Clunes, J. ${ }^{a^{*}}$, Navarro, J. ${ }^{a}$, Pinochet, D. ${ }^{a}$

${ }^{a}$ Facultad de Ciencias Agrarias, Instituto de Ingeniería Agraria y Suelos, Universidad Austral de Chile. Independencia 641, Valdivia, Chile.
A B S T R A C T

The aim of this study was to determine the relationship between the management system of agricultural residues and organic matter in two volcanic soils of Southern Chile. The soil samples were collected in areas where there are mixed of volcanic materials, and this was evaluated on the extractable aluminum content variability. When this parameter was related to soil organic matter content allowed us to confirm that the extractable aluminum is the most important index of the soil organic matter value, explaining organic matter level by more than $60 \%$ and suggesting that residues management would explain up to $40 \%$ of total organic matter content. No relation was found between extractable aluminum and exchangeable aluminum, or a correlation between organic matter content and mineral nitrogen in the soil samples.

In general, grassland management systems allow an accumulation of organic matter content and conversely, crops, mainly grain crops are more extractive management system since they use the nutrients and reduce the organic matter content of the soil. It was established that both the mineral nitrogen and soluble carbon not have a significant correlation with the levels of organic matter in the soil.
*Corresponding author:

Dante Pinochet

E-mail address:

dpinoche@uach.cl

johnclunes@gmail.com

\title{
RESUMEN
}

El objetivo de este estudio fue determinar la relación existente entre el tipo de manejo agrícola y los contenidos de materia orgánica presente en los suelos volcánicos de la zona Sur de Chile. Las muestras se colectaron en sectores con suelos mezclados de distintos materiales volcánicos, lo que afectó los resultados esperados. Esto se hizo evidente al observar la variabilidad de los contenidos de aluminio extractable de las muestras de suelo. Los resultados muestran que este índice estuvo relacionado con la variación de los valores de materia orgánica del suelo, permitiendo explicar los niveles de materia orgánica en más de un $60 \%$, lo que indicaría que el manejo de residuos solo estaría influyendo hasta en un 40\% del total de la materia orgánica del suelo. No se pudo establecer relaciones entre el aluminio extractable y el aluminio intercambiable, así como tampoco entre los contenidos de materia orgánica y nitrógeno mineral presente en el perfil del suelo.

El manejo agrícola de praderas permite, en general, una acumulación de los contenidos de materia orgánica y por el contrario, los cultivos, principalmente los cultivos de granos, son manejos más extractivos, dado que utilizan los nutrientes y reducen el contenido de materia orgánica presente en el suelo. De los parámetros de fertilidad medidos en este estudio se pudo establecer que tanto el nitrógeno mineral así como el carbono soluble no presentan una correlación significativa con los niveles de materia orgánica presentes en el suelo.

Palabras clave: Índice de aluminio extractable, materia orgánica, nitrógeno mineral, manejo de residuos.

\section{INTRODUCCIÓN}

La materia orgánica del suelo (MOS) es un parámetro determinado por controles bióticos, como la abundancia producción y tipos de especies de plantas y de la producción microbial y por controles ambientales, tales como la temperatura, contenido de agua y la textura del suelo (Sollins et al., 1996). La dinámica y preserva- ción de la MOS es crucial porque mejora la estructura y porosidad del suelo, su fertilidad y, por ende, la producción de los cultivos (Denef et al., 2004). El mantenimiento de la materia orgánica, a través de un manejo integrado de la fertilidad del suelo, es importante para la calidad del suelo y la persistencia de la diversidad de la fauna del suelo y biomasa microbial (Ayuke et al., 2011). El uso de monocultivos sin la incorporación de 
residuos, en conjunto con el uso de fertilizantes artificiales para compensar los nutrientes perdidos, ha sido una de las causas más comunes a las que se le adjudica la disminución de los niveles de MOS (Körschens et al., 1998). Laird y Chang (2013) demostraron la importancia crítica de los residuos en el mantenimiento de los niveles de nitrógeno (N) y carbono orgánico (CO) del suelo independientes del sistema de manejo. Además, se conoce (Six et al., 1999) que la labranza generalmente reduce la agregación y el contenido de partículas de materia orgánica (PMO) y que los agregados del suelo son sensibles a las prácticas de manejo, donde el incremento de la desintegración de los agregados debido a la labranza pueden dar lugar a un aumento en la descomposición de la MO (Six et al., 1998). Kong et al. (2005) corroboran que la tasa de cambio en el carbono orgánico del suelo (COS) está directamente relacionada con el nivel de entrada de carbono (C) a partir de residuos de cultivos y enmiendas que añaden C. Por lo tanto, es importante aumentar, como fertilizante, el uso de materiales orgánicos como el estiércol, la incorporación de los residuos vegetales y/o las rotaciones dentro de un sitio específico y definir el papel que tiene las praderas en el aumento de la materia orgánica del suelo.

De este modo, el objetivo de este estudio fue evaluar el rol que tienen las praderas en la restauración de la MOS y cómo el manejo de cultivo sin una adecuada incorporación de residuos disminuye el contenido de MOS. Para esto se evaluó, en dos profundidades $(0-10 \mathrm{~cm}$ y $10-20 \mathrm{~cm}$ ) la variación del contenido de material orgánico de acuerdo a 9 años consecutivos de manejo de cultivo y 6 años consecutivos de manejo de pradera en sitios de características climáticas similares, en suelos volcánicos con variación en parámetros que reflejan la reactividad, la condición de suelo y su fertilidad.

\section{MATERIAL Y MÉTODO}

\section{Descripción de las muestras de suelo}

Las muestras fueron colectadas desde dos predios ubicados en suelos volcánicos de la Región de los Lagos y de la Región de los Ríos de Chile (Figura 1). Estas muestras se asocian a 2 series de suelo, la serie de suelo Los Lagos con pendientes dominantes que varían entre $4-15 \%$, suelos profundos, moderadamente estructurados, con una fracción arcillosa constituida por materiales amorfos, especialmente alofán, con densidades aparentes promedios de 0,77 y reales de 2,19, con una porosidad total de $65 \%$. La materia orgánica se encuentra en niveles muy altos en los horizontes superficiales y va disminuyendo en profundidad, siendo determinante en los valores encontrados de porosidad total, constantes hídricas, humedad aprovechable y densidad aparente. Los suelos de esta serie son fuertemente ácidos en superficie y se hacen ligeramente áci- dos en profundidad. Son bajos en potasio de intercambio, fósforo aprovechable y nitrógeno total. Con niveles de materia orgánica altos en horizontes superficiales y con predominancia de arcillas amorfas. La segunda serie es Osorno con pendientes dominantes de 2 a 5\%; bien estructurados, con una característica discontinuidad litológica, la que se evidencia tanto en el análisis de las arcillas como en el de las arenas, con densidad aparente y real con 0,61 y 2,25 respectivamente, siendo su porosidad total de $73 \%$. Los niveles de materia orgánica son muy altos en superficie y disminuyen gradualmente en profundidad. Son suelos fuertemente ácidos en superficie y se hacen neutros en profundidad. En general, se trata de suelos que poseen bajos niveles de elementos nutritivos, con valores extremadamente bajos de fósforo aprovechable, situación que se agudiza en los horizontes inferiores debido a que la capacidad de fijación de fósforo y el aluminio y fierro se incrementan a valores extremadamente (IREN-UACH, 1978).

Las muestras de suelos fueron tomadas a dos profundidades $(0-10 \mathrm{~cm}$ y $10-20 \mathrm{~cm})$ con tres repeticiones. En cada predio se seleccionaron sitios sometidos a dos historiales de manejo distintos en relación con el ingreso de residuos (praderas y cultivos) y su variabilidad temporal hasta 10 años de pradera y 6 años de cultivos continuos, para determinar la relación existente entre historial de manejo de residuos y la materia orgánica presente en el suelo. Las muestras compuestas de 10 sub-muestras desde sectores representativos de cada sitio y en tres repeticiones por cada profundidad de suelo, resultando en un total de 90 muestras.

\section{Preparación de las muestras de suelo}

Las muestras fueron secadas al aire $\left(30^{\circ} \mathrm{C}\right.$ por una semana) y tamizadas a $2 \mathrm{~mm}$. El manejo de residuos en los potreros de cultivos (Cuadro 1) mayoritariamente fueron quemados y llevados a cenizas o en otras sistemas sometidos a barbecho químico, para el posterior establecimiento de nuevos cultivos o praderas.

\section{Determinación de parámetros de fertilidad y condición de suelo en las muestras}

Se determinó el carbono total del suelo por digestión húmeda y titulación, el nitrógeno mineral disponible (nitratos más amonio) por extracción con KCl 2 M (determinación por arrastre de vapor), el aluminio intercambiable en $\mathrm{KCl} 1 \mathrm{M}$ y el aluminio extractable con acetato de amonio $1 \mathrm{M}$ a pH 4,8, la medición de $\mathrm{pH}$ fue en agua en relación 1:2,5 Sadzawka et al. (2006). La determinación del carbono soluble se realizó por extracción, digestión y titulación (Anderson y Ingram, 1993).

Las muestras fueron analizadas como muestras al azar, donde el tratamiento es años de cultivo y pradera con tres repeticiones en cada profundidad y se efectua- 
A)

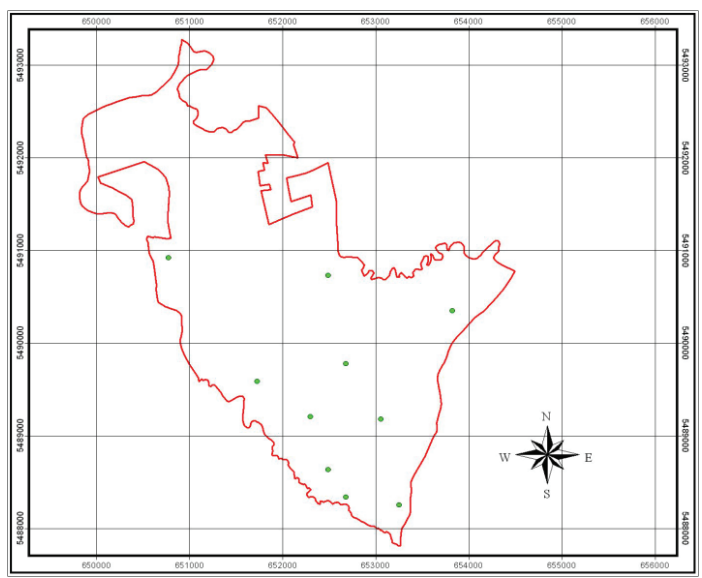

B)

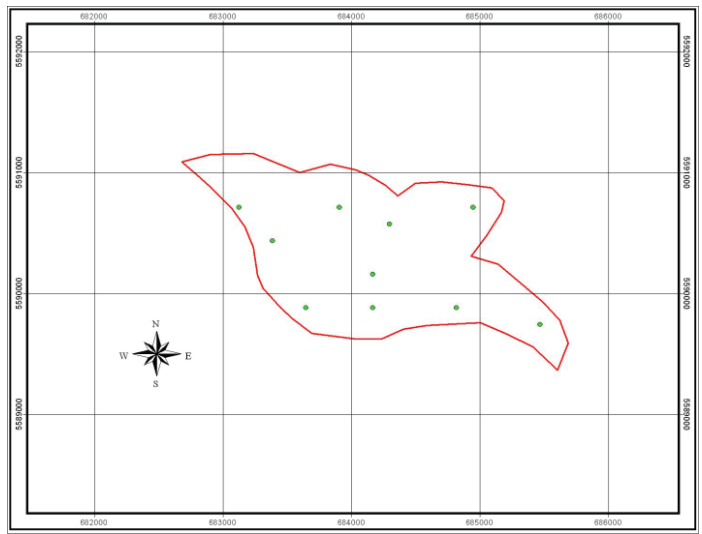

Figura 1. Ubicación geográfica de los predios y sectores de muestreo en estos. A: Predio Región de Los Lagos; B: Predio Región de Los Ríos.

Figure 1. Geographic location of the farms and sampling areas within them. A: Los Lagos Region farm; Los Ríos Region farm.

ron un test de medias (Tukey) y análisis de regresión para analizar las tendencias de acumulación o desacumulación a través de los años de cultivo o pradera. Para esto se utilizaron los softwares estadísticos Statgraphics 2.0 y GraphPad Prism 4.0.

\section{RESULTADOS Y DISCUSIÓN}

El análisis químico de los parámetros de fertilidad relevantes para evaluar su variación en el tiempo, en un sistema de manejo constante a diferentes profundidades se muestra en los cuadros 2 y 3 .

\section{Relaciones entre los parámetros analizados}

La dinámica de la materia orgánica del suelo está determinada en gran medida por el tipo de suelo (Six et al., 1998). El aluminio extractable es un parámetro que per- mite evaluar al tipo de suelo, dada la relación existente entre su contenido y el tipo de arcilla en suelos volcánicos (Rodríguez, 1993; Huygens et al., 2005; Matus et al., 2008).

Los valores de Al extractable determinan que las muestras analizadas (cultivos y praderas) no corresponden a los mismos suelos, aunque correspondían a los mismos predios (Figura 2). Esto se debería a que las Series de suelo volcánico presentan variaciones con inclusiones de Series vecinas de origen diverso. En general, los valores de $\mathrm{Al}$ extractable son mayores en las muestras correspondientes a una profundidad de muestreo de 10-20 cm, lo que ha sido observado comúnmente en estos suelos (IREN-UACH, 1978).

El valor de $\mathrm{Al}$ extractable en el suelo volcánico A (SVA) con manejo de praderas fue aproximadamente 2 veces mayor que el valor de suelo volcánico B (SVB) con manejo de cultivos y ubicados en sectores simila- 
Cuadro 1: Historial de manejos agrícolas en praderas y cultivos de los suelos.

Table 1: Record of soil management practices in pastures and crops.

\begin{tabular}{|c|c|}
\hline $\begin{array}{l}\text { Años de } \\
\text { manejo }\end{array}$ & $\begin{array}{l}\text { Historial de manejo suelo volcánico A } \\
\text { (SVA Pradera) }\end{array}$ \\
\hline 1 & $\begin{array}{l}\text { Pradera establecida por primera vez, fertilizada con } \\
100 \mathrm{~kg} \text { de Supernitro } 27 \text { ( } 24 \text { unidades de nitrógeno) } \\
\text { y } 320 \mathrm{~kg} \text { de SPT (126 unidades de fosforo) en Marzo, } \\
\text { además de una aplicación de } 150 \mathrm{~kg} \text { de urea ( } 138 \text { uni- } \\
\text { dades de nitrógeno) en Agosto (Fertilización base). }\end{array}$ \\
\hline 2 & $\begin{array}{l}\text { Segundo año de pradera, con aplicaciones anuales de } \\
\text { fertilización base. }\end{array}$ \\
\hline 3 & $\begin{array}{l}\text { Tercero año de pradera, posterior a un cultivo de ce- } \\
\text { bada, con aplicaciones anuales de fertilización base y } \\
300 \mathrm{~kg} \text { de yeso en Noviembre. }\end{array}$ \\
\hline 4 & $\begin{array}{l}\text { Cuarto año de pradera, regenerada el tercer año, con } \\
\text { aplicaciones anuales de fertilización base. }\end{array}$ \\
\hline 5 & $\begin{array}{l}\text { Quinto año de pradera, después de un cultivo de ave- } \\
\text { na-lupino, y regenerada el tercer año, con aplicacio- } \\
\text { nes anuales de fertilización base. }\end{array}$ \\
\hline 6 & $\begin{array}{l}\text { Sexto año de pradera, regenerada el tercer y cuarto } \\
\text { año. Fertilizaciones de SPT ( } 320 \mathrm{~kg}) \text {, Supernitro (100 } \\
\text { kg), y en el año } 2001 \text { Mezcla SQM } 3225 \text { (700 kg). }\end{array}$ \\
\hline 7 & $\begin{array}{l}\text { Séptimo año de pradera, regenerada el segundo y } \\
\text { cuarto año. Fertilizada con urea }(100 \mathrm{~kg}) \text {, Supernitro } \\
(100 \mathrm{~kg}) \text { y SPT }(350 \mathrm{~kg}) \text {, en distintos años. }\end{array}$ \\
\hline 8 & $\begin{array}{l}\text { Octavo año de pradera, regenerada el segundo y ter- } \\
\text { cer año. Fertilizada con mezcla SQM } 3225 \text { y Superni- } \\
\text { tro. }\end{array}$ \\
\hline 9 & $\begin{array}{l}\text { Noveno año de pradera, sobre residuos de maíz, rege- } \\
\text { nerada el segundo, cuarto y quinto año. Fertilizacio- } \\
\text { nes anuales con Supernitro, parcializado en } 2 \text { dosis de } \\
100 \text { y } 150 \mathrm{~kg} \text {. }\end{array}$ \\
\hline 10 & $\begin{array}{l}\text { Decimo años de pradera, regenerada al segundo, } \\
\text { cuarto, quinto y sexto año. Fertilizaciones similares a } \\
\text { los potreros anteriores, además de hydrosulfan en los } \\
\text { años de regeneración ( } 300 \mathrm{~kg} \text { ). }\end{array}$ \\
\hline
\end{tabular}

Años de manejo (SVB Cultivo)

\section{Historial de manejo suelo volcánico $\mathrm{A}$}

Historial de manejo suelo volcánico B (SVC Pradera)
Pradera establecida por primera vez, fertilizada con 200 unidades de N-urea (100 Otoño y 100 Primavera), 60 unidades de K-Muriato y 112 unidades de $\mathrm{P}_{2} \mathrm{O}_{5}$.

Segundo año de pradera, fertilizada con 200 unidades de N-urea (100 Otoño y 100 Primavera), 60 unidades de K-Muriato y 112 unidades de $\mathrm{P}_{2} \mathrm{O}_{5}$ anualmente.

Tercero año de pradera, fertilizada con 200 unidades de N-urea (100 Otoño y 100 Primavera), 60 unidades de K-Muriato y 112 unidades de $\mathrm{P}_{2} \mathrm{O}_{5}$ anualmente.

Cuarto año de pradera, fertilizada con 200 unidades de N-urea (100 Otoño y 100 Primavera), 60 unidades de K-Muriato y 112 unidades de $\mathrm{P}_{2} \mathrm{O}_{5}$ anualmente.

Quinto año de pradera, fertilizada con 200 unidades de N-urea (100 Otoño y 100 Primavera), 60 unidades de K-Muriato y 112 unidades de $\mathrm{P}_{2} \mathrm{O}_{5}$ anualmente.

Sexto año de pradera, fertilizada con 200 unidades de N-urea (100 Otoño y 100 Primavera), 60 unidades de K-Muriato y 112 unidades de $\mathrm{P}_{2} \mathrm{O}_{5}$ anualmente.

Séptimo año de pradera, fertilizada con 200 unidades de N-urea (100 Otoño y 100 Primavera), 60 unidades de K-Muriato y 112 unidades de $\mathrm{P}_{2} \mathrm{O}_{5}$ anualmente.

Octavo año de pradera, fertilizada con 200 unidades de N-urea (100 Otoño y 100 Primavera), 60 unidades de K-Muriato y 112 unidades de $\mathrm{P}_{2} \mathrm{O}_{5}$ anualmente.

Noveno año de pradera, fertilizada con 200 unidades de N-urea (100 Otoño y 100 Primavera), 60 unidades de K-Muriato y 112 unidades de $\mathrm{P}_{2} \mathrm{O}_{5}$ anualmente.
Primer año de trigo después de pradera

2 Segundo año de trigo sobre trigo.

3

Primer año de nabo después de 2 años de trigo consecutivo.

4 Primer año de trigo después de 1 año de avena y 2 de trigo.

5

Primer año de avena sobre nabo, con 3 años anteriores de trigo.

6
Historial de manejo suelo volcánico B (SVD Cultivo)

Primer año con trigo, fertilizado con 240 unidades de $\mathrm{P}$, 150 unidades de $\mathrm{K}$ - muriato y nitrógeno parcializado en 200 unidades como urea y 20 unidades como Nitromag (Fertilización base).

Segundo año con trigo, fertilizado con Fertilización base.

Tercer año de trigo consecutivo, fertilizado con Fertilización base.

Cuarto año de trigo fertilizado con Fertilización base.

Primer año de avena después de 4 años de trigo, fertilizado con Fertilización base. 
Cuadro 2. Análisis químico de los suelos a dos profundidades, evaluados en el tiempo.

Table 2: Chemical analysis of soils at two depths through time.

\begin{tabular}{|c|c|c|c|c|c|c|}
\hline \multicolumn{7}{|c|}{ Suelo volcánico A (Pradera, 0 - $10 \mathrm{~cm}$ ) } \\
\hline Tiempo & $\begin{array}{c}\text { Materia } \\
\text { orgánica } \\
(\%)\end{array}$ & $\begin{array}{c}\text { Carbono } \\
\text { soluble } \\
(\mathrm{ppm})\end{array}$ & $\begin{array}{l}\text { Nitrógeno } \\
\text { (mg/kg) }\end{array}$ & $\begin{array}{c}\text { Aluminio } \\
\text { intercambiable } \\
\left(\mathrm{cmol}_{+} / \mathrm{kg}\right)\end{array}$ & $\begin{array}{c}\text { Aluminio } \\
\text { extractable } \\
(\mathrm{mg} / \mathrm{kg})\end{array}$ & $\begin{array}{c}\text { pH } \\
(1: 2,5) \\
\end{array}$ \\
\hline Año 1 & 20,74 & 413 & 18,43 & 0,113 & 1209 & 5,83 \\
\hline Año 2 & 20,41 & 413 & 23,10 & 0,119 & 1278 & 5,83 \\
\hline Año 3 & 19,40 & 453 & 24,50 & 0,168 & 1319 & 5,67 \\
\hline Año 4 & 19,03 & 453 & 33,37 & 0,102 & 998 & 5,57 \\
\hline Año 5 & 18,02 & 453 & 34,07 & 0,302 & 1179 & 5,47 \\
\hline \multicolumn{7}{|c|}{ Suelo volcánico B (Cultivo, 0 - $10 \mathrm{~cm}$ ) } \\
\hline Año 1 & 14,21 & 413 & 42,47 & 0,297 & 567 & 5,37 \\
\hline Año 2 & 11,77 & 536 & 36,40 & 0,151 & 458 & 5,32 \\
\hline Año 3 & 10,62 & 578 & 21,23 & 0,174 & 502 & 5,65 \\
\hline Año 4 & 10,25 & 578 & 20,10 & 0,211 & 1128 & 5,23 \\
\hline Año 5 & 10,48 & 536 & 18,53 & 0,119 & 281 & 5,57 \\
\hline \multicolumn{7}{|c|}{ Suelo volcánico A (Pradera, $10-20 \mathrm{~cm}$ ) } \\
\hline Año 1 & 20,05 & 289 & 17,27 & 0,097 & 1290 & 5,77 \\
\hline Año 2 & 19,17 & 289 & 14,70 & 0,100 & 1342 & 5,77 \\
\hline Año 3 & 18,07 & 289 & 18,20 & 0,095 & 1327 & 5,83 \\
\hline Año 4 & 16,51 & 371 & 20,53 & 0,053 & 1256 & 5,90 \\
\hline Año 5 & 17,20 & 454 & 17,27 & 0,185 & 1560 & 5,63 \\
\hline \multicolumn{7}{|c|}{ Suelo volcánico B (Cultivo, 10 - $20 \mathrm{~cm}$ ) } \\
\hline Año 1 & 11,31 & 536 & 25,20 & 0,244 & 691 & 5,51 \\
\hline Año 2 & 9,79 & 595 & 21,23 & 0,252 & 527 & 5,25 \\
\hline Año 3 & 8,60 & 536 & 25,90 & 0,298 & 578 & 5,42 \\
\hline Año 4 & 7,13 & 660 & 15,67 & 0,190 & 962 & 5,40 \\
\hline Año 5 & 7,63 & 536 & 13,53 & 0,290 & 393 & 5,37 \\
\hline
\end{tabular}

res. Por su parte, el valor de Al extractable del suelo volcánico D (SVD) con manejo de cultivos fue el doble del valor de suelo volcánico C (SVC) con manejo de praderas y ubicados en sectores similares entre ellos.

\section{Relación entre el aluminio extractable y la materia orgánica}

Hassink (1997) ha postulado que el contenido de arcilla y posteriormente Percival et al. (2000) el tipo de arcilla, son determinantes en el contenido de materia orgánica en los suelos (MOS), ya que los microagregados $<2 \mathrm{~mm}$ son sitios de protección a la descomposición de la MOS, tanto por atrapamiento como por la adsorción (Chenu y Plante, 2006). Varios estudios
(Percival et al., 2000; Huygens et al., 2005; Jansen et al., 2011; Matus et al., 2008) han mostrado una relación entre el contenido de $\mathrm{Al}$ extractable con pirofosfato y en oxalato con el contenido de MOS, lo que ratifica que en los suelos volcánicos es el Al extractable un parámetro indicador del tipo de arcilla presente, que es relacionado con la MOS (Cuadro 4). Chevallier et al. (2010) determinaron que el contenido de carbono orgánico del suelo (COS) se correlacionó significativamente con el contenido de alofán en el horizonte de $0-20 \mathrm{~cm}$, confirmando que los suelos alofánicos contienen hasta tres veces más COS que suelos no alofánicos. Por tal razón, ellos sugieren que la alta estabilización del COS en Andisoles no es sólo el resultado de una adsorción de COS en superficies minerales, sino también el resultado de 
Cuadro 3: Análisis químico de los parámetros de fertilidad de los suelos a dos profundidades de muestreo.

Table 3: Chemical analysis of fertility parameters of soils at two sampling depths.

\begin{tabular}{|c|c|c|c|c|c|c|}
\hline \multicolumn{7}{|c|}{ Suelo volcánico C (Pradera, 0 - $10 \mathrm{~cm}$ ) } \\
\hline Tiempo & $\begin{array}{l}\text { Materia } \\
\text { orgánica }\end{array}$ & $\begin{array}{c}\text { Carbono } \\
\text { soluble }\end{array}$ & Nitrógeno & $\begin{array}{c}\text { Aluminio } \\
\text { intercambiable }\end{array}$ & $\begin{array}{l}\text { Aluminio } \\
\text { extractable }\end{array}$ & $\mathrm{pH}_{\mathrm{agua}}$ \\
\hline & (\%) & $(\mathrm{ppm})$ & (mg/kg) & $\left(\mathrm{cmol}_{+} / \mathrm{kg}\right)$ & $(\mathrm{mg} / \mathrm{kg})$ & $(1: 2,5)$ \\
\hline Año 1 & 12,00 & 495 & 30,10 & 0,181 & 1257 & 6,03 \\
\hline Año 2 & 15,82 & 578 & 21,00 & 0,251 & 954 & 5,80 \\
\hline Año 3 & 17,93 & 536 & 24,27 & 0,086 & 1133 & 5,86 \\
\hline Año 4 & 17,98 & 454 & 24,50 & 0,088 & 1106 & 5,86 \\
\hline Año 5 & 17,47 & 454 & 22,87 & 0,072 & 1076 & 5,47 \\
\hline \multicolumn{7}{|c|}{ Suelo volcánico D (Cultivo, 0 - $10 \mathrm{~cm}$ ) } \\
\hline Año 1 & 24,05 & 784 & 68,83 & 0,255 & 2033 & 5,38 \\
\hline Año 2 & 21,10 & 784 & 106,87 & 0,277 & 2350 & 5,24 \\
\hline Año 3 & 20,97 & 578 & 91,93 & 0,227 & 1848 & 5,37 \\
\hline Año 4 & 21,47 & 536 & 106,40 & 0,260 & 1876 & 5,03 \\
\hline Año 5 & 22,71 & 619 & 125,77 & 0,239 & 1987 & 4,51 \\
\hline \multicolumn{7}{|c|}{ Suelo Volcánico C (Pradera, $10-20 \mathrm{~cm}$ ) } \\
\hline Año 1 & 10,21 & 330 & 17,27 & 0,036 & 1355 & 6,16 \\
\hline Año 2 & 11,45 & 495 & 14,23 & 0,183 & 1146 & 5,80 \\
\hline Año 3 & 13,06 & 536 & 19,60 & 0,025 & 1516 & 6,14 \\
\hline Año 4 & 14,57 & 413 & 14,00 & 0,060 & 1569 & 5,90 \\
\hline Año 5 & 14,44 & 454 & 14,10 & 0,052 & 1437 & 5,53 \\
\hline \multicolumn{7}{|c|}{ Suelo Volcánico D (Cultivo 10 - $20 \mathrm{~cm}$ ) } \\
\hline Año 1 & 19,22 & 825 & 62,07 & 0,188 & 2189 & 5,49 \\
\hline Año 2 & 19,26 & 701 & 57,40 & 0,200 & 2146 & 5,25 \\
\hline Año 3 & 19,31 & 660 & 61,37 & 0,200 & 2209 & 5,40 \\
\hline Año 4 & 20,46 & 660 & 63,70 & 0,240 & 2261 & 5,23 \\
\hline Año 5 & 22,44 & 619 & 125,30 & 0,234 & 2091 & 4,60 \\
\hline
\end{tabular}

Cuadro 4: Valores promedios de materia orgánica y aluminio extractable en los cuatro suelos y sus manejos.

Table 4: Mean values of organic matter and extractable aluminium in the four soils and their managements.

\begin{tabular}{ccc}
\hline Suelo y manejo & Materia orgánica & Aluminio extractable \\
\hline Suelo volcánico A - Pradera & $\mathbf{( g / 1 0 0 g )}$ & $\mathbf{( m g / \mathbf { k g } )}$ \\
Suelo volcánico B - Cultivo & $18,21 \pm 1,5$ & $1406 \pm 208$ \\
Suelo volcánico C - Pradera & $9,80 \pm 2,1$ & $592 \pm 225$ \\
Suelo volcánico D - Cultivo & $14,92 \pm 2,5$ & $1413 \pm 160$ \\
\hline
\end{tabular}




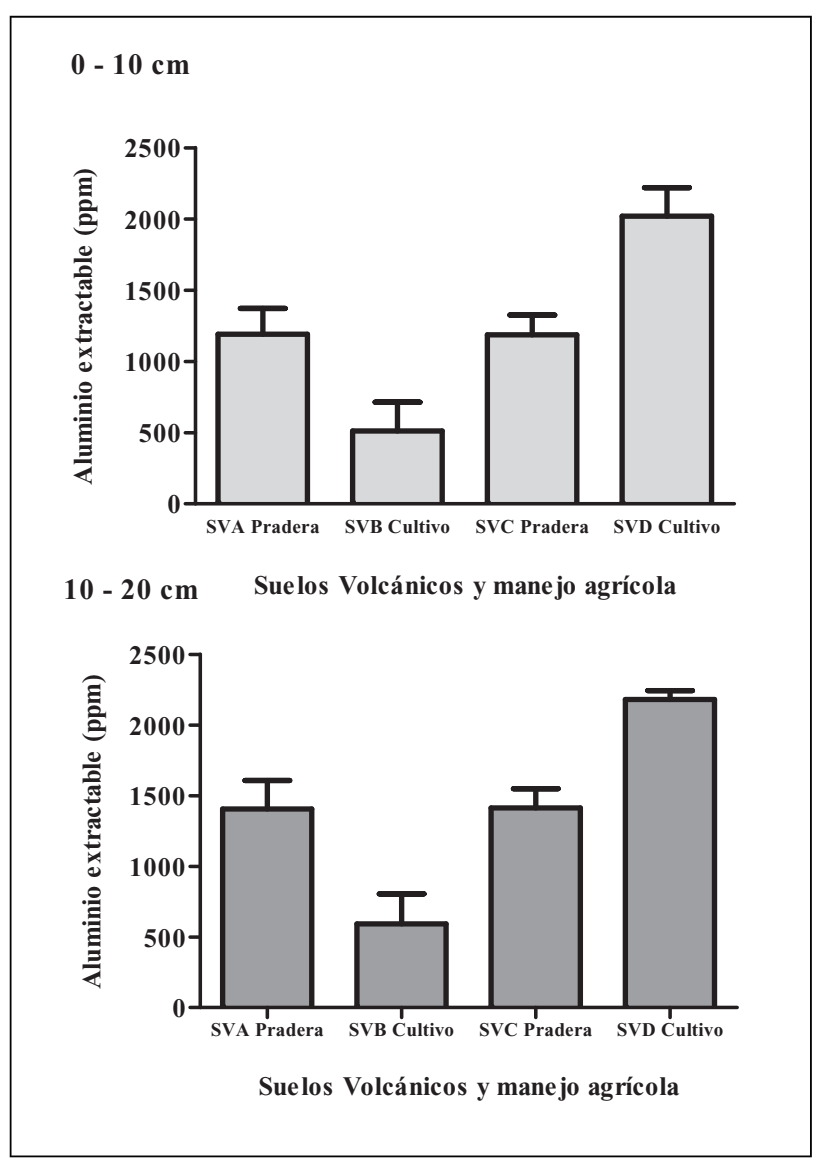

Figura 2: Valores de aluminio extractable según el manejo agrícola en los suelos volcánicos evaluados.

Figure 2: Extractable aluminium values according to agricultural management in the evaluated volcanic soils.

una mala accesibilidad de COS en la estructura de mesoporos. Los valores determinados en el presente estudio muestran que la mayor variación de la MOS está asociada con la variación del contenido de Al extractable del suelo en una misma profundidad y, en el mismo sitio, con la profundidad de muestreo. Kögel-Knabner et al. (2008) determinaron que la cantidad y el porcentaje relativo de COS que se encuentra en las fracciones órgano-minerales es muy variable entre los tipos de suelo y entre los horizontes dentro del mismo suelo. En los resultados que se presentan, la variación de la MOS con la profundidad del suelo no está relacionada con el aumento del Al extractable en profundidad (cuadros 2 y 3). Ello sugiere que los sitios reactivos del suelo serían mayores en la medida que no están saturados con materia orgánica y que la capacidad de retención de aquella recién adicionada (residuos) sería mayor en profundidad que en la capa superficial, que ha recibido históricamente un mayor contenido de residuos orgánicos. De esta forma, en los horizontes inferiores el grado de estabilización de la MOS en términos de ad- sorción a la fase mineral de suelos sería más relevante que el horizonte superficial (Rumpel et al., 2012).

Las relaciones determinadas entre el contenido de $\mathrm{Al}$ extractable y MOS, independiente del manejo realizado al suelo, se ajustaron a una regresión exponencial del tipo $\mathrm{Y}=\mathrm{Ymax}(1-\exp (-\mathrm{kX}))$. Las ecuaciones ajustadas fueron significativas y presentaron un $\mathrm{R}^{2}$ de 0,653 y 0,637 y un error estándar (Sy.x) de 2,29\% MO y 2,60\% M0, para la profundidad de $0-10 \mathrm{~cm}$ y $10-20 \mathrm{~cm}$ (Figura 3).

Los resultados muestran que, en términos generales, el contenido de MOS en primer lugar obedece al tipo y reactividad de las arcillas (Al extractable) más que al manejo agrícola en los suelos. Esto es apoyado por Garrido y Matus (2012), quienes muestran que el $\mathrm{Al}$ extractable en acetato de amonio ácido fue el factor principal que explica la variación del COS en suelos similares, en lugar de las variables climáticas y el contenido de arcilla. De esta forma, la tendencia general, es que a mayor contenido de $\mathrm{Al}$ extractable, mayor es el contenido de MOS. Sin embargo, el factor de determinación de las ecuaciones fue cerca del $64 \%$, indicando que este no es el único parámetro que determina el contenido de MOS (Six et al., 2000; Krull et al., 2003; Kögel-knabner et al., 2008). Por ello se supone que el manejo estaría determinando sólo parcialmente el contenido de MOS.

\section{Relación entre aluminio extractable e intercambiable de los suelos}

El Al intercambiable es un parámetro asociado con la acidez del suelo, lo cual es una condición del suelo. Por su parte, el Al extractable es un parámetro que está relacionado con el tipo de reactividad de las arcillas presente en suelos volcánicos. Por ello, ambos parámetros no deberían estar relacionados (Jansen et al., 2011); esto es, a mayor acidez no necesariamente existe un mayor contenido de $\mathrm{Al}$ extractable aunque si de $\mathrm{Al}$ intercambiable. En este sentido, la Figura 4 muestra este hecho a las dos profundidades evaluadas, ratificándose que la acidez del suelo es independiente de los niveles de $\mathrm{Al}$ extractable.

\section{Relación entre el Al intercambiable y la materia orgánica}

Los resultados de este estudio arrojaron que no existe una relación entre los niveles de $\mathrm{Al}$ intercambiable y la MOS presente en las muestras evaluadas (Figura 5), al considerar los manejos bajo los cuales se encuentra sometidas las muestras, ni la profundidad de muestreo.

\section{Relación entre la materia orgánica y el nitrógeno mineral del suelo}

Es común que se plantee que a mayor MOS debería haber más $\mathrm{N}$ disponible. Diversos autores (Zagal et al., 


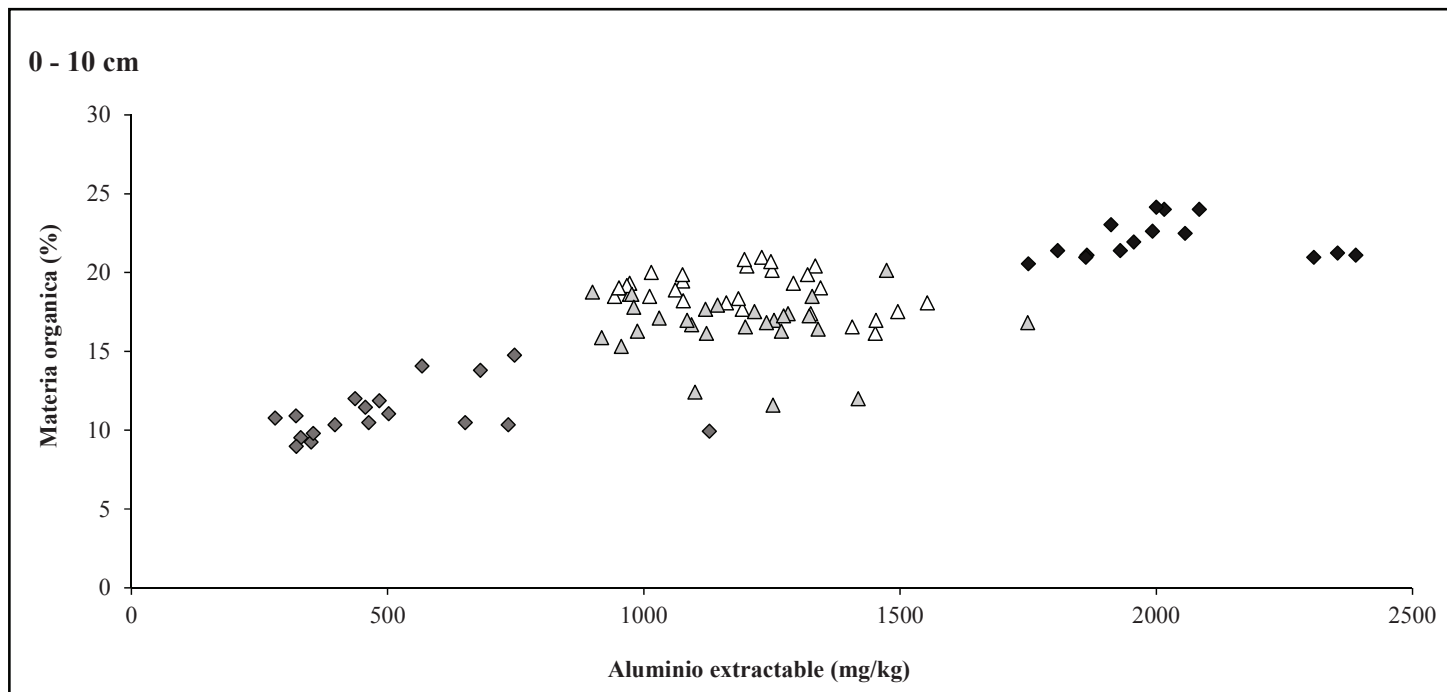

$10-20 \mathrm{~cm}$

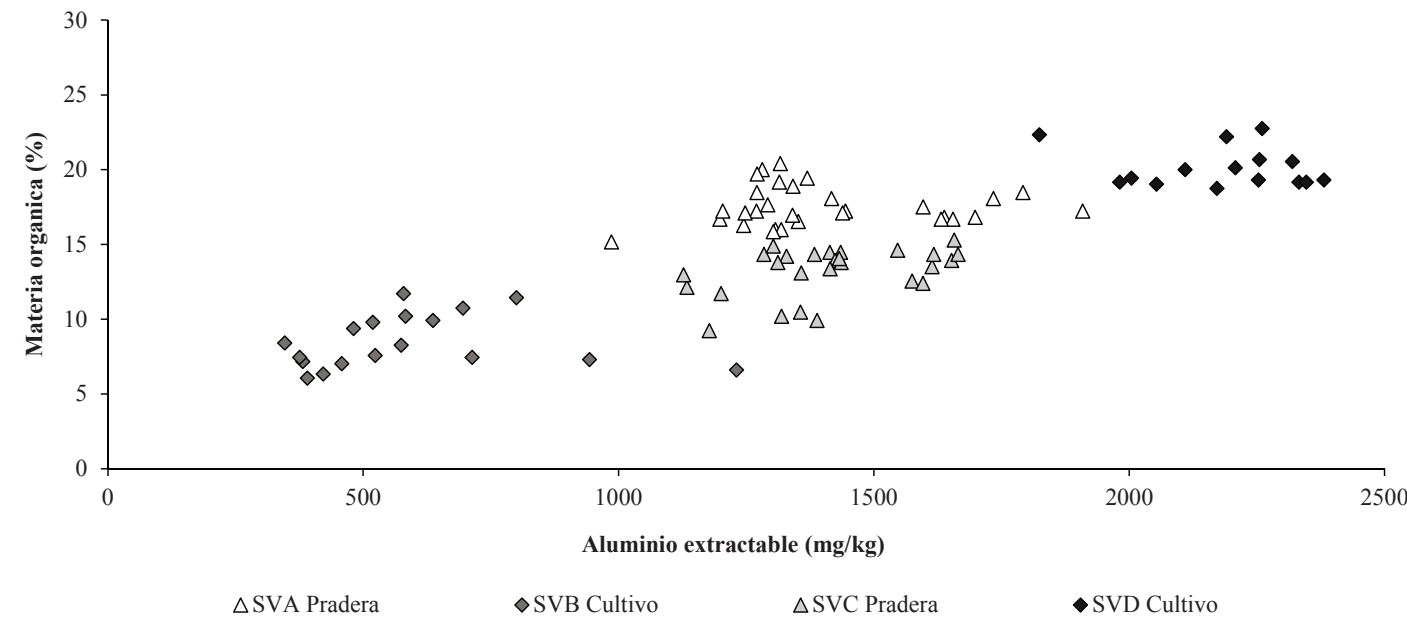

Figura 3: Relación entre el aluminio extractable y la materia orgánica presente en cada suelo y profundidad de muestreo.

Figure 3: Relationship between extractable aluminium and organic matter in each soil and sampling depth.

2002; Wanek et al., 2010) han descrito la importancia e influencia de la vida microbial y cómo ésta interviene en los procesos degradativos que dan origen a las transformaciones de nitrógeno en el suelo. Según la Figura 6, se observa que para ambos suelos no existió una buena relación entre ambas variables, lo cual es corroborado por los bajos coeficientes de determinación $(0,23$ y 0,26 para profundidades de $0-10 \mathrm{~cm}$ y $10-20 \mathrm{~cm}$, respectivamente).

\section{Evaluación del contenido de materia orgánica con el historial de manejo}

Existen dos factores de influyen en el contenido de MOS: (a) el tipo fracción arcillosa presente en la mues- tra de suelo y (b) el sistema de manejo de ingreso de residuos (cantidad de residuos en la pradera o cultivo) en los suelos analizados a través del tiempo. El manejo agrícola, sobre el suelo a través del tiempo, influye en la concentración de MOS y aumenta su reciclaje en diferentes grados (Balesdent et al., 2000; Gong et al., 2009). En este estudio al igual que en Zagal et al. (2002) y Apezteguia y Sereno (2002), se trató de aislar el factor clima utilizando sectores de diferentes manejos ubicados en la misma localidad y presentes en suelos ligeramente ácidos (IREN-UACH, 1978).

Se consideraron dos tendencias de la evolución de la MOS dependiendo del manejo. Es así como para el manejo en el SVA, la pradera presentó una acumulación de MOS a través del tiempo, mostrando valores relativa- 


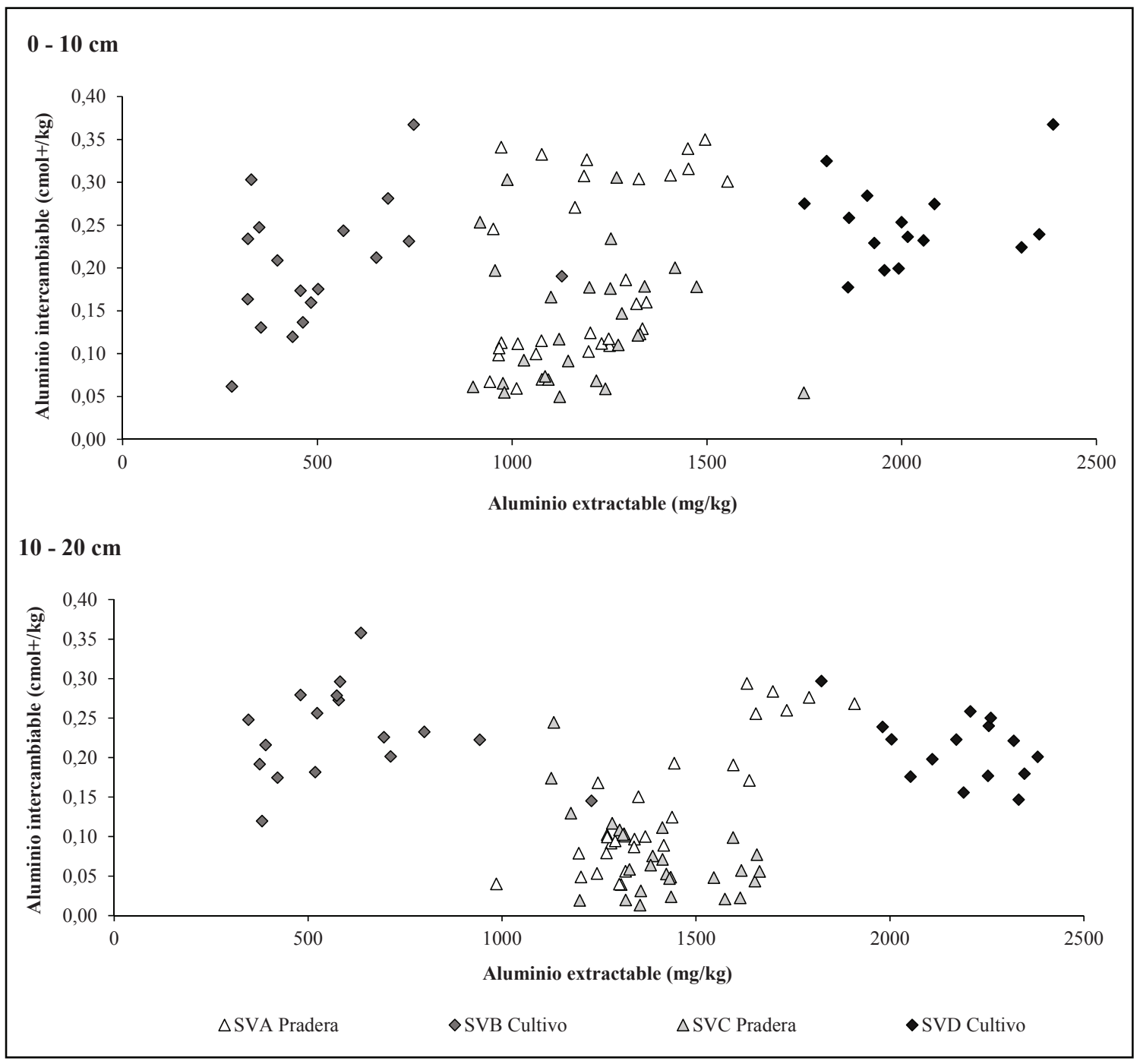

Figura 4: Relación entre el aluminio extractable y el intercambiable de cada suelo y profundidad de muestreo.

Figure 4: Relationship between extractable and interchangeable aluminium in each soil and sampling depth.

mente constante (Figura 7). Para el SVB bajo un historial de cultivo se observa una disminución del contenido de MOS en la medida que transcurren los años de cultivo, indicando que habría habido un menor ingreso de residuos (usualmente rastrojo de cereales), debido a su quema.

La pérdida de MOS del SVB de cultivo en este estudio fue mucho mayor o más pronunciada en los primeros años bajo este sistema, haciéndose más estable y menos agresiva con el paso del tiempo donde estas pérdidas tienden a estabilizarse. En este sentido varios autores (Vilches et al., 2002; Zagal et al., 2002; Mikha y Rice, 2004; Zotarelli et al., 2005) han comparado la evolución de diversos factores en el suelo a través del tiempo bajo sistemas de pradera y cultivo, pudiendo observar que los contenidos de CO total disminuyeron notoriamente en el suelo con el cambio de uso (de pra- dera a cultivo), especialmente en el primer y segundo año. A pesar de ello, Laird y Chang (2013) indican que la eliminación de residuos tiene un impacto sustancial en los niveles de CO del suelo, demostrando la importancia crítica que los residuos tiene en el mantenimiento de estos niveles independientemente del sistema de manejo, ya que sus resultados indican una degradación sustancial de la calidad del suelo como consecuencia de 19 años de la eliminación del 90\% de los residuos. Por tal razón, la labranza acelera la descomposición de la MOS y destruye los agregados estables (Gong et al., 2009; Martins et al., 2009).

Para el caso del SVC pradera y SVD cultivo existe una situación diametralmente opuesta, presentando una acumulación bajo ambos manejos (Figura 7). Estas variaciones tuvieron una relación directa con los valores obtenidos de $\mathrm{Al}$ extractable. Los materiales arcillo- 


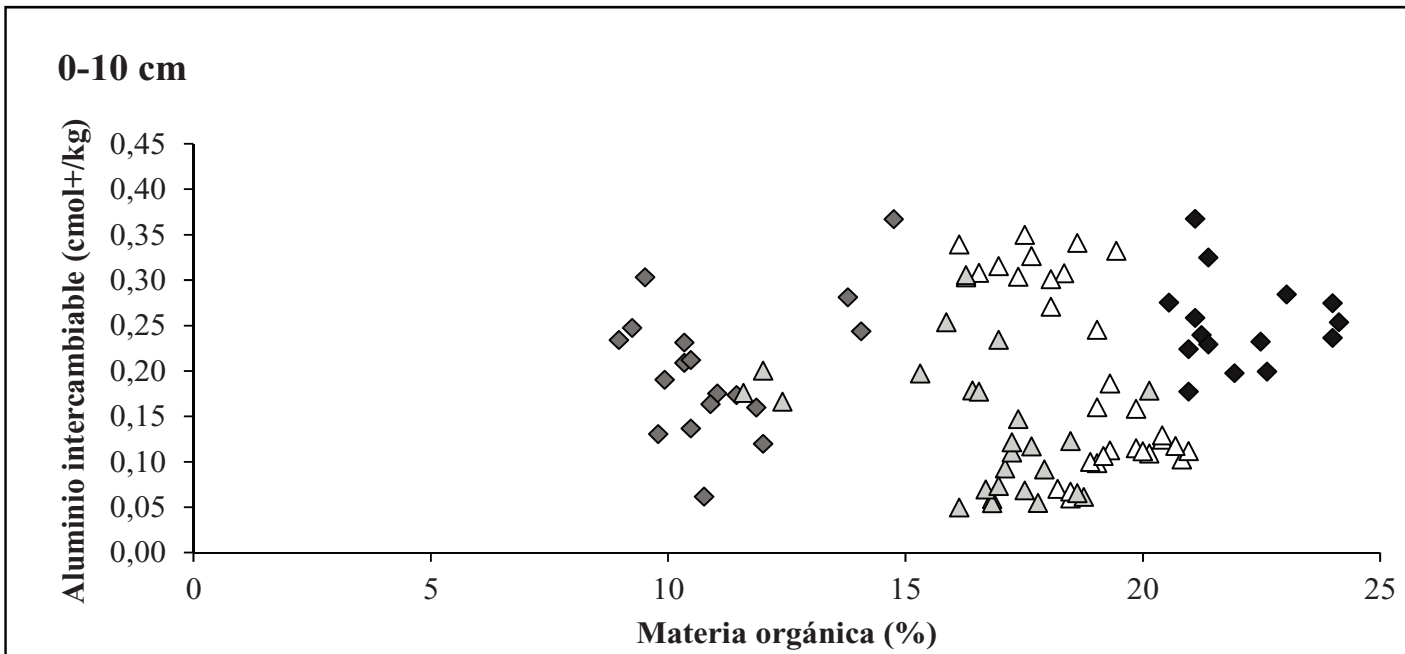

$10-20 \mathrm{~cm}$

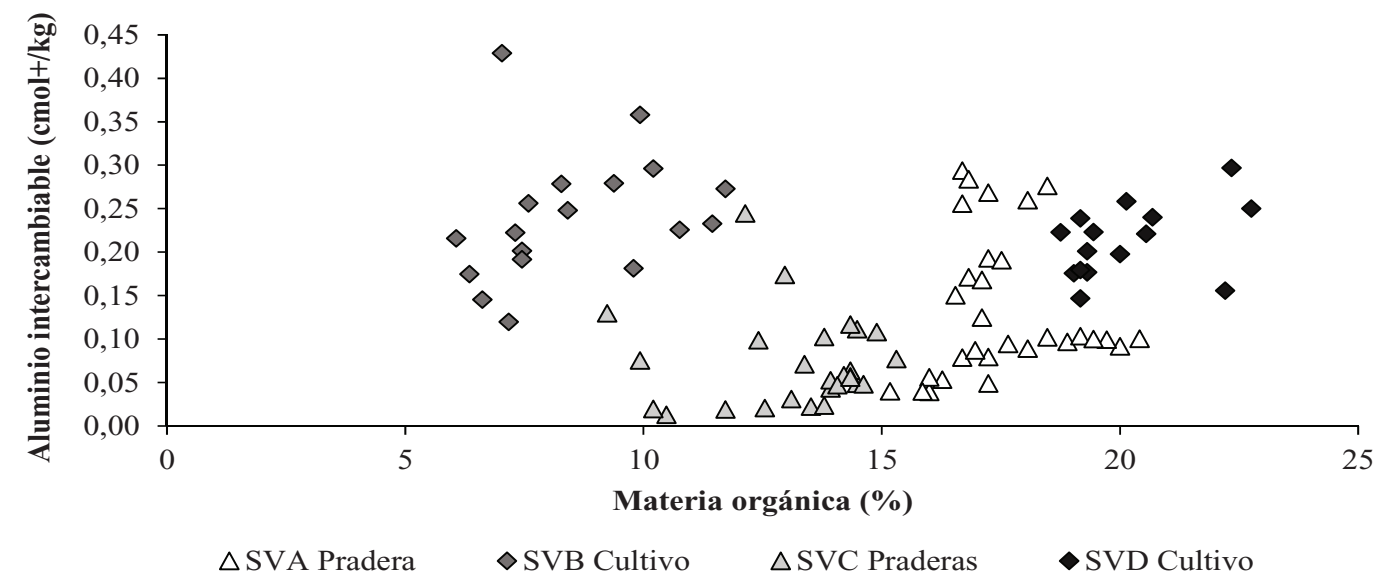

Figura 5: Relación entre el aluminio intercambiable y la materia orgánica en cada suelo y profundidad de muestreo.

Figure 5: Relationship between interchangeable aluminium and organic matter in each soil and sampling depth.

so serían el principal factor que controla la estabilización de MOS en los suelos alofánicos de Chile (Garrido y Matus, 2012). Los valores de MOS y su tendencia como era de esperar presentaron las mayores cantidades a menor profundidad de muestreo, relación obtenida y descrita con anterioridad por Apezteguía y Sereno (2002) y Gong et al. (2009). Esta diferencia se hace más estrecha bajo el manejo de cultivo, lo que se puede explicar por la inversión constante del suelo, llevando los residuos vegetales a mayores profundidades (Pinochet et al., 2006). Además existe una acumulación de esta MOS por parte de la pradera, diferenciándose del caso anterior en que al ocurrir la transición hacia el manejo de cultivo no se afectó dicha acumulación perdiendo sus niveles; por el contrario, la tendencia se mantuvo, observándose incluso un gran incremento en los valores en el primer año, y posterior a esto se mantuvo la acumulación a una tasa relativamente constante. Esta situación fue observada por Whitmore et al. (2012), los cuales determinan que el COS no aumenta o disminuye a una velocidad lineal en respuesta a un cambio en el manejo, pero se mueve de un nivel de equilibrio a otro durante un período de años. El valor de equilibrio real es una función del tipo de suelo, clima, sistema de cultivo, aportes de materia orgánica y de una serie de factores de manejo, definiéndose un equilibrio entre las tasas de insumos orgánicos y descomposición. Además, estos resultados son explicados en parte por los valores de $\mathrm{Al}$ extractable que presentan las muestras las cuales presentan la misma tendencia y, en menor grado, porque la pradera presentaría mayores contenidos de biomasa microbial. Esto afectaría directamente los contenidos de MOS, obteniéndose valores más bajos en las concentraciones de CO de estas praderas y valores mayores en las rotaciones de cultivos, lo que sería similar a los resultados obtenidos por Zagal et al. (2002). 


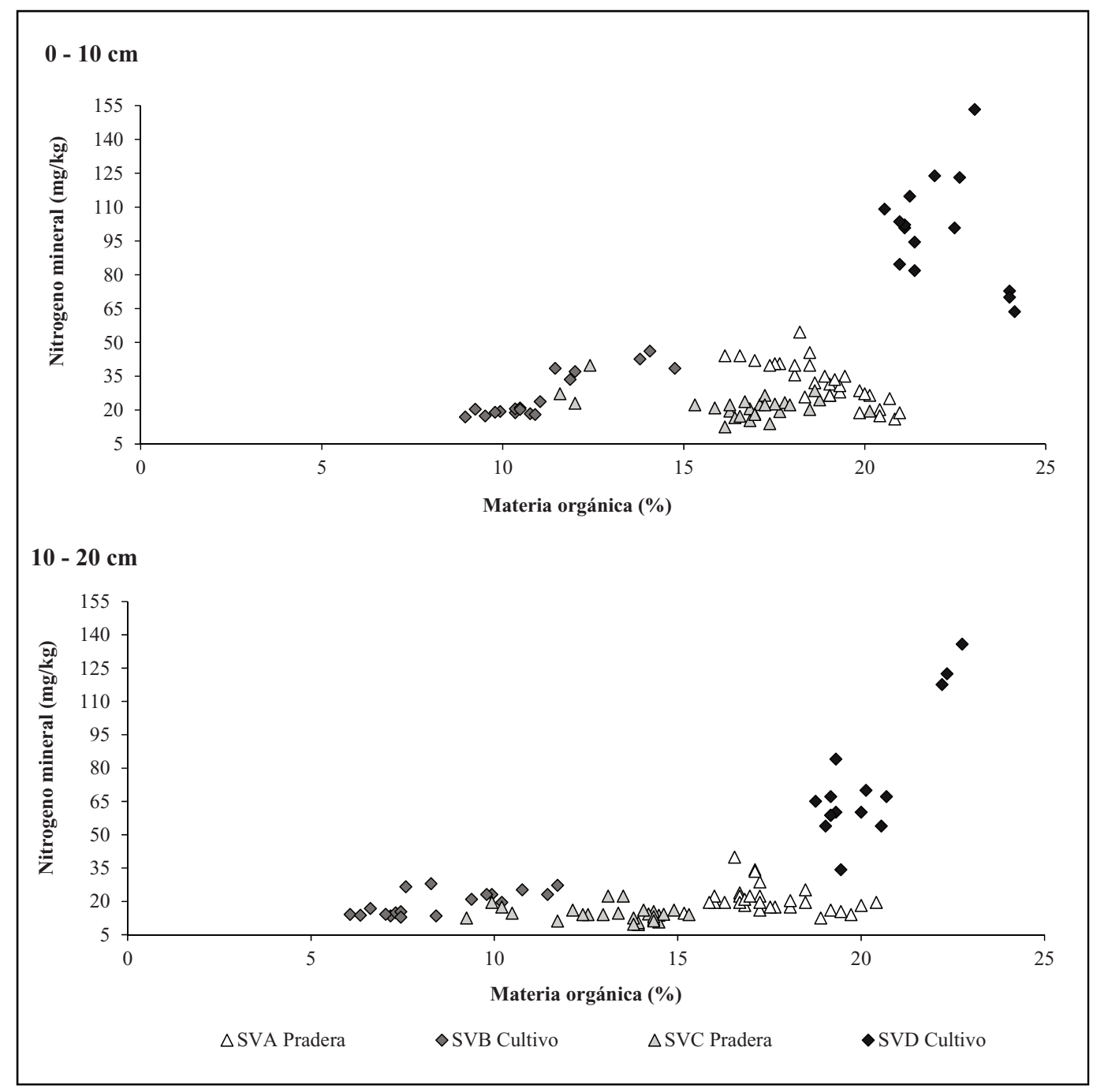

Figura 6: Relación entre el nitrógeno y la materia orgánica de cada suelo y profundidad de muestreo.

Figure 6: Relationship between nitrogen and organic matter in each soil and sampling depth.

El Cuadro 5 permite observar que existen diferencias estadísticas altamente significativas de MOS en los suelos (P-valor $<0,01$ ), por efecto del manejo en ambas profundidades de muestreo.

Todos los suelos, independientes de su manejo, presentan valores más altos de materia orgánica en las muestra de $0-10 \mathrm{~cm}$ de profundidad, que de 10-20 $\mathrm{cm}$, lo cual coincide con lo expuesto por Mikha y Rice (2004). Estas diferencias a distintas profundidades fueron observadas también por otros autores (Six et al., 2000; Apezteguía y Sereno 2002; Moore y Turunen, 2004; Dalzell et al., 2013), concluyendo que la cantidad de MOS disminuye en profundidad dentro del perfil. Para el caso del SVA pradera, el porcentaje de MOS encontrado es mayor, en ambas profundidades, que el SVC pradera. Del mismo modo, el porcentaje de MOS encontrado en SVD cultivo es mayor en ambas profundidades que el SVB cultivo. Contrario a lo esperado, el SVD cultivo presentó porcentajes mayores de MOS en ambas profundidades, con diferencias altamente significativas, que los demás suelos (SVD cultivo > SVA pradera $>$ SVB pradera $>$ SVC cultivo). Esto se debe principalmente al tipo de arcilla del suelo y el contenido de MOS recalcitrante, ya que existen claras diferencias entre los distintos tipos de suelo con respecto al tipo de $\mathrm{MO}$ en asociaciones órgano-minerales (Kögel-Knabner et al., 2008). Esto se apoya en el estudio de Chenu y Plante (2006) donde determinaron que la protección de la MO por adsorción debe ser poco afectada por el cultivo, ya que el éste no cambia la textura del suelo y tampoco la mineralogía. Por otro lado, la labranza convencional resulta en una disminución de MOS en los primeros $30 \mathrm{~cm}$ del suelo, por el contrario para sistemas sin labranza en los primeros $30 \mathrm{~cm}$ del suelo, la masa de MOS aumenta pro- 


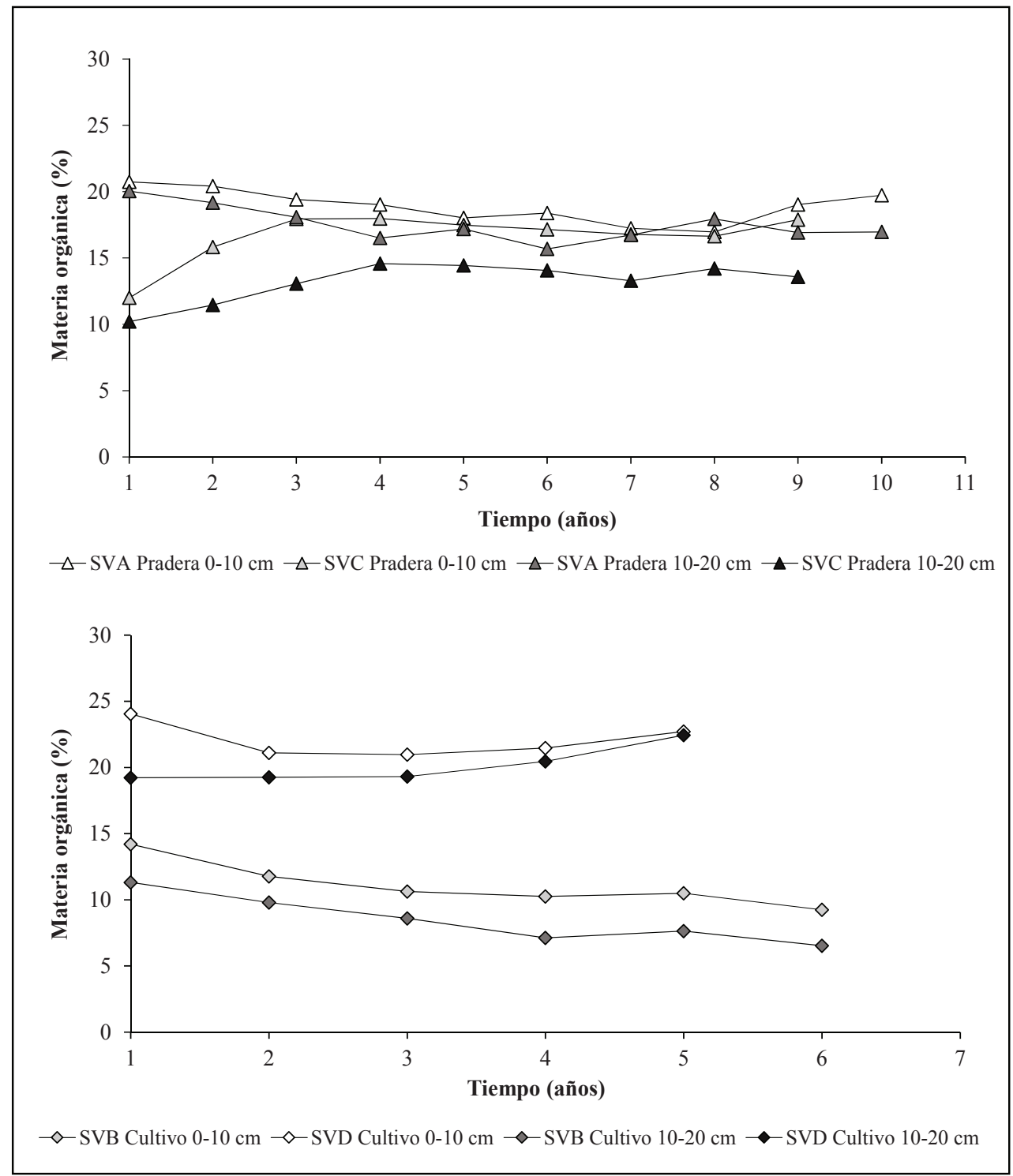

Figura 7: Evolución en la materia orgánica para ambos suelos a través del tiempo según tipo de manejo y profundidad de muestreo.

Figure 7: Evolution of organic matter for both soils through time according to type of management and sampling depth.

Cuadro 5: Niveles de materia orgánica en los suelos a dos profundidades de muestreo, determinados en el tiempo.

Table 5: Soil organic matter levels at two sampling depths through time.

\begin{tabular}{ccc}
\hline & \multicolumn{2}{c}{ Materia orgánica (\%) } \\
\cline { 2 - 3 } Suelos & \multicolumn{2}{c}{ Profundidad de muestreo } \\
\cline { 2 - 3 } & $0-10 \mathrm{~cm}$ & $10-20 \mathrm{~cm}$ \\
\hline Suelo volcánico A - Pradera & $19,52 \pm 0,26 \mathrm{a}$ & $18,19 \pm 0,36 \mathrm{a}$ \\
Suelo volcánico B - Cultivo & $11,47 \pm 0,41 \mathrm{~b}$ & $8,88 \pm 0,43 \mathrm{~b}$ \\
Suelo volcánico C - Pradera & $16,24 \pm 0,62 \mathrm{~b}$ & $12,74 \pm 0,50 \mathrm{~b}$ \\
Suelo volcánico D - Cultivo & $22,06 \pm 0,32 \mathrm{a}$ & $20,14 \pm 0,34 \mathrm{a}$ \\
\hline
\end{tabular}

Letras distintas denotan diferencias altamente significativas $(\mathrm{P}<0,01)$ prueba de Tukey $(5 \%)$. 
porcionalmente a la cantidad de residuos incorporados del cultivos (Dalzell et al., 2013). Cuando se compara manejo de praderas versus cultivos se encuentra que en la primera, por haber un menor movimiento del suelo, existe una mayor acumulación de MOS, debido a un reciclaje más efectivo. Plaza et al. (2013), sugieren que las fracciones de MOS, bajo sistemas de no labranza, son más altos que los del suelo con labranza convencional y que la protección física de los microagregados contribuyen a la estabilización a largo plazo en sistemas de no labranza, proporcionando protección física no sólo de MO-mineral libre, sino también a MO protegida químicamente por adsorción sobre las superficies minerales. Por lo tanto, la MOS se encuentra en constante flujo en diferentes pools dependiendo de la asociación físicoquímico que presentan con dichos sitios.

\section{CONCLUSIONES}

La variable de suelo que estuvo más relacionada con el contenido de materia orgánica de los suelos (MOS) es su contenido de aluminio (Al) extractable, sugiriendo que ésta se correlaciona más con el tipo y contenido de arcilla de los suelos volcánicos que otras variables evaluadas. De esta forma las muestras de suelo con mayores niveles de $\mathrm{Al}$ extractable coincidieron con los valores más altos de MOS. Como primera aproximación se sugiere que el contenido de Al extractable en suelos volcánicos explica hasta en un 64\% su contenido de MOS.

No se determinó una relación los niveles de Al extractable y Al intercambiable para los suelos evaluados bajo los dos sistemas de manejo de residuos. Situación similar se determinó en la relación entre el Al intercambiable con los valores de MOS. Existió además una relación entre $\mathrm{Al}$ intercambiable y los valores de $\mathrm{pH}$ de las muestras de suelo, lo que sugiere que la condición de acidez no explica la diferente acumulación de MOS. Tampoco se obtuvo una relación estrecha entre el contenido de MOS y los valores de nitrógeno $(\mathrm{N})$ mineral, lo que sugiere que la mineralización del $\mathrm{N}$ no está determinada directamente por el contenido de materia orgánica en los suelos volcánicos estudiados.

$\mathrm{Al}$ evaluar el efecto del manejo de residuos (incorporación o ausencia) a través del historial de manejo de los suelos y sistemas de manejo, se muestra que bajo historial de praderas la MOS tiende a mantenerse en el tiempo, en tanto que ésta tiende a decrecer en el caso de los cultivos. La medición de carbono soluble realizada en este estudio no mostró una correlación consistente con el historial de manejo tanto para pradera como para cultivo.

\section{REFERENCIAS}

ANDERSON, J.M., INGRAM, J.S.I. 1993. Tropical soil biology and fertility. A handbook of methods. Wallingford.
APEZTEGUIA, H., SERENO, R. 2002. Influencia de los sistemas de labranza sobre la cantidad y calidad del carbono orgánico del suelo. Agricultura Técnica 62 (3): 418-426.

AYUKE, F.O., BRUSSAARD, L., VANLAUWE, B., SIX, J., LELEI, D.K., KIBUNJA, C.N., PULLEMAN, M.M. 2011. Soil fertility management: Impacts on soil macrofauna, soil aggregation and soil organic matter allocation. Applied Soil Ecology 48: 53-62.

BALESDENT, J., CHENU, C., BALABANE, M. 2000. Relationship of soil organic matter dynamics to physical protection and tillage. Soil and Tillage Research 53: 215-230.

CHENU, C., PLANTE, A.F. 2006. Clay-sized organo-mineral complexes in a cultivation chronosequence: revisiting the concept of the 'primary organo-mineral complex'. European Journal of Soil Science 57: 596-607.

CHEVALLIER, T., WOIGNIER, T., TOUCET, J., BLANCHART, E. 2010. Organic carbon stabilization in the fractal pore structure of Andosols. Geoderma 159: 182-188.

DALZELL, B.J., JOHNSON, J.M.F., TALLAKSEN, J., ALLAN, D., BARBOUR, N.W. 2013. Simulated impacts of crop residue removal and tillage on soil organic matter maintenance. Soil Science Society of American Journal 77 (4): 1349-1356.

DENEF, K., SIX, J., MERCKX, R., PAUSTIAN, K. 2004. Carbon sequestration in microaggregates of No-tillage soils with different clay mineralogy. Soil Science Society of American Journal 68: 1935-1944.

GONG, Y., CHEN, H., HOU, R., LI, H., FAN, M., KUZYAKOV, Y. 2009. Effects of 11 years of conservation tillage on soil organic matter fractions in wheat monoculture in Loess Plateau of China. Soil and Tillage Research 106: 85-94.

HASSINK, J. 1997. The capacity of soils to preserve organic C and $\mathrm{N}$ by their association with clay and silt particles. Plant Soil 191: 77-87.

HUYGENS, D., BOECKX, P., VAN CLEEMPUT, O., GODOY, R., OYARZÚN, C. 2005. Aggregate structure and stability linked to carbon dynamics in a south Chilean Andisol. Biogeosciences 2: 203-238.

INSTITUTO NACIONAL DE INVESTIGACIÓN DE RECURSOS NATURALES (IREN) - CORFO - UNIVERSIDAD AUSTRAL DE CHILE. 1978. Estudio de suelos de la provincia de Valdivia. Santiago.

JANSEN, B., TONNEIJCK, F. H., VERSTRATEN, J. M. 2011. Selective extraction methods for aluminium, iron and organic carbon from Montane volcanic ash soils. Pedosphere 21(5): 549-565.

KONG, A., SIX, J., BRYANT, D., FORD DENISON, R., VAN KESSEL, C. 2005. The relationship between carbon input, aggregation, and soil organic carbon stabilization in sustaina- 
ble cropping systems. Soil Science Society of American Journal 69: 1078-1085.

KÖGEL-KNABNER. I., GUGGENBERGER, G., KLEBER, M., KANDELER, E., KALBITZ, K., SCHEU, S., EUSTERHUES, K., LEINWEBER, P. 2008. Organo-mineral associations in temperate soils: Integrating biology, mineralogy, and organic matter chemistry. Journal Plant Nutrition Soil Science 171: 61-82.

KÖRSCHENS, M., WEIGEL, A. AND SCHULZ, E. 1998. Turnover of soil organic matter (SOM) and long-term balances - tools for evaluating sustainable productivity of soils. Journal of Plant Nutrition and Soil Science 161: 409-424.

KRULL, E.S., BALDOCK, J.A., SKJEMSTAD, J.O. 2003. Importance of mechanisms and processes of the stabilisation of soil organic matter for modelling carbon turnover. Functional Plant Biology 30: 207-222

LAIRD, D., CHANG, C.W. 2013. Long-term impacts of residue harvesting on soil quality. Soil and Tillage Research 134: 33-40.

MARTINS, M., CORA, J.E., FALQUETO R.J., VALENTE A.M. 2009. Crop type influences soil aggregation and organic matter under no-tillage. Soil and Tillage Research 104: 2229.

MATUS, F., GARRIDO, E., SEPULVEDA, N., CARCAMO, I., PANICHINI, M., ZAGAL, E. 2008. Relationship between extractable $\mathrm{Al}$ and organic $\mathrm{C}$ in volcanic soils of Chile. Geoderma 148: 180-188.

GARRIDO, E., MATUS. F. 2012. Are organo-mineral complexes and allophane content determinant factors for the carbon level in Chilean volcanic soils? Catena 92: 106-112.

MIKHA, M., RICE, C. 2004. Tillage and manure effects on soils and aggregate-associated carbon and nitrogen. Soil Science Society of American Journal 68: 809-816.

MOORE, T., TURUNEN, J. 2004. Carbon accumulation and storage in mineral subsoil beneath peat. Soil Science Society of America Journal 68: 690-696.

PERCIVAL, H.J., PARFITT, R.L., SCOTT, N.A. 2000. Factors controlling soil carbon levels in New Zealand grasslands: Is clay content important? Soil Science Society of America Journal 64:1623-1630.

PINOCHET, D., MENDOZA, J., GALVIS, A. 2006. Potencial de mineralización de nitrógeno de un Hapludand con distintos manejos agrícolas. Ciencia e Investigación Agraria 27(2): 97-106.

PLAZA, C., COURTIER-MURIAS, D., FERNÁNDEZ, J.M., POLO, A., SIMPSON, A.J. 2013. Physical, chemical, and biochemical mechanisms of soil organic matter stabilization under conservation tillage systems: A central role for microbes and microbial by-products in $\mathrm{C}$ sequestration. Soil Biology and Biochemistry 57: 124-134.

RODRÍGUEZ, J. 1993. La fertilización de los cultivos: un método racional. Facultad de Agronomía. Pontificia Universidad Católica de Chile. Santiago.

RUMPEL, C., RODRÍGUEZ, A., GONZÁLEZ, J.A., ARBELO, C. CHABBI, A., NUNAN, N., GONZÁLEZ, F.J. 2012. Contrasting composition of free and mineral-bound organic matter in top- and subsoil horizons of Andosols. Biology and Fertility of Soil 48: 401-411.

SADZAWKA, A., CARRASCO, M.A., GREZ, R., MORA, M.L., FLORES, H., NEAMAN, A. 2006. Métodos de análisis recomendados para los suelos de Chile. Centro regional de investigación La Platina. Santiago.

SIX, J., ELLIOTT, E.T., PAUSTIAN, K., DORAN, J.W. 1998. Aggregation and soil organic matter accumulation in cultivated and native grassland soils. Soil Science Society of American Journal 62: 1367-1377.

SIX, J., ELLIOTT, E.T., PAUSTIAN, K. 1999. Aggregate and soil organic matter dynamics under conventional and notillage systems. Soil Science Society of American Journal 63(5): 1350-1358.

SIX, J., ELLIOTT, E.T., PAUSTIAN, K. 2000. Soil macroaggregate turnover and microaggregate formation: a mechanism for $\mathrm{C}$ sequestration under no-tillage agriculture. Soil Biology and Biochemistry 32: 2099-2103.

SOLLINS, P., HOMANN, P., CALDWELL B. A. 1996. Stabilization and destabilization of soil organic matter: mechanisms and controls. Geoderma 74: 65-105.

VILCHES, M. S., ALZUGARAY, C., MONTICO, S. 2002. Efecto de la labranza y duración de las praderas sobre la condición física de un suelo Argiudol Vértico de Argentina. Ciencia e Investigación Agraria 29(3): 159-169.

WANEK, W., INSELSBACHER, E., UMANA, N., STANGE, F., GORFER, M., SCHÜLLER, E., RIPKA, K., ZECHMEISTER-BOLTENSTERN, S., HOOD-NOVOTNY, R., STRAUSS, J. 2010. Short-term competition between crop plants and soil microbes for inorganic N fertilizer. Soil Biology and Biochemistry $42: 360-372$.

WHITMORE, A.P., POWLSON, D.S., BHOGAL, A., CHAMBERS, B.J., COLEMAN, K., MACDONALD, A.J GOULDING, K.W.T. 2012. The potential to increase soil carbon stocks through reduced tillage or organic material additions in England and Wales: A case study. Agriculture, Ecosystems and Environment 146: 23-33.

ZAGAL, E., RODRIGUEZ, N., VIDAL, I., FLORES, B. 2002. La fracción liviana de la materia orgánica de un suelo volcánico bajo distintos manejos agronómicos como índice de cambios de la materia orgánica lábil. Agricultura Técnica 62(2): 284-296. 